\title{
Orientational ordering of nanorods of different length in diblock copolymers
}

\author{
M. A. Osipov ${ }^{1,2}$, Y. V. Kudryavtsev², A. S. Ushakova ${ }^{2}$, and A. V. Berezkin ${ }^{2}$ \\ 1 Department of Mathematics and Statistics, \\ University of Strathclyde, Glasgow G1 $1 X H$, Scotland, UK \\ 2 Topchiev Institute of Petrochemical Synthesis, \\ Russian Academy of Sciences, 119991 Moscow, Russia
}

\begin{abstract}
Orientational and positional ordering of nanorods in the lamellae phase of diblock copolymers has been investigated using a simple theoretical model and dissipative dynamics simulations. Orientational order parameter and local concentration profiles of nanorods are calculated numerically and extracted from computer simulations data for different values of the nanoparticle length and different number of the interaction sites in the model nanorod. The predictions of the molecular theory are compared with the results of computer simulations.. It has been found that the nanorods are orientationally ordered in the boundary region between the domains and the orientational order parameter changes its sign at the domain wall. At the same time there exists some quantitative discrepancy between theory and computer simulations which is partially removed when a similar model of a nanorod is employed both in the molecular theory and in coarse-grained molecular dynamics simulations.
\end{abstract}




\section{INTRODUCTION}

Liquid crystal nanocomposites are based on nematic and smectic LC phases doped by isotropic or anisotropic nanoparticles of various chemical structure [1] In particular, nematic LC nanocomposites are considered to be promising materials as they are characterised by improved switching voltages and switching times [2-6] and enhanced values of the dielectric susceptibility if ferroelectric nanoparticles are used [8, 12-14]. Nanoparticles (NPs) may effect the stability of the nematic phase depending on their shape and size. Indeed it has been shown that the nematic-isotropic (N-I) transition temperature is decreased when the LC material is doped with isotropic nanoparticles [15-18] while strongly anisotropic NPs may increase the N-I transition temperature [7-9, 19]. These effects have been interpreted theoretically using a molecular theory [10, 20].

Polymer nanocomposites also attract significant attention as NPs substantially improve various properties of block copolymers [21-27]. However, some of the most interesting physical effects are related to the orientational ordering of nanorods at the boundaries between blocks. Polymer material in the bulk of every block is isotropic and hence the nanorods may orientationally order only in the interfacial regions. This effect has recently been described theoretically using a simple model which accounts for different interactions between the NP and the monomers of the two different kinds [32]. In the boundary region the effective interaction of a NP with all neighboring monomers becomes anisotropic and aligns the nanoparticles. The orientational ordering of nanorods in diblock copolymers has indeed been observed experimentally. Metal nanorods with different functional groups are aligned parallel to the domain walls $[28,31]$ while semiconducter nanorods are ordered perpendicular to the cylindrical domain walls. [29, 30].

Orientational ordering of anisotropic nanoparticles in the lamellae and hexagonal phases has recently been studied theoretically using the approximation of the perfect phase separated structure of the polymer host matrix. [32]. NPs have been assumed to possess spherical shape but the interaction potential between NPs and monomers is composed of both isotropic and the anisotropic parts. It has been shown that anisotropic NPs are aligned parallel to the domain wall in one of the blocks and perpendicular to the wall in the neighboring block behind the wall. Orientational order parameter profiles have been calculated numerically. 
The main results of Ref. [32] have recently been verified by molecular dynamics simulation [33] of block copolymers doped with nanorods. Rigid anisotropic nanoparticles have been modelled by a string of spheres interacting with the monomers of both kinds, and dissipative particle dynamics has been used to evaluate spatial distribution of both monomers and the nanoparticles and the orientational order parameter profiles for different values of the model parameters. The simulations confirm the orientational order of nanorods and the sign inversion of the orientational order parameter in the boundary region. However, simulations reveal that, in contrast to the molecular theory, the nanorods are ordered both in the boundary region and in the bulk of the domains and the nematic order parameter is higher than in the molecular theory.

The agreement between theory and computer simulations has been significantly improved in our recent paper [34] where a different model of a nanoparticle has been used. The nanorod is composed of the two equal spheres separated by a distance $l$ which interact with the monomers in the two domains. As a result the effective interaction between such a nanorod and a monomer is anisotropic and explicitly depends on the nanoparticle length similar to computer simulations. Such a molecular theory yields sufficiently high values of the orientational order parameter and a broader distribution of NPs in the interfacial region. However, the discrepancy between theory and experiment is still too large, and in this paper we consider the ordering of much longer nanorods composed of five interaction spheres which closely mimics the structure of NPs employed in simulations. The corresponding concentration and order parameter profiles are calculated numerically and compared with the ones obtained for short nanorods composed of two interaction spheres and with the results of computer simulations. One notes that high values of the orientational order parameter of the nanorods in polymer nanocomposites may lead to large dielectric anisotropy which, in principle, enables one to align block copolymer nanocomposites using external electric field. At present the alignment of block copolymers is a complicated problem which is far from being completely solved.

The paper is arranged as follows. In Section 2 we summarise the results of the molecular field theory of polymer nanocomposites and derive explicit expressions for the nematic order parameter and the concentration distribution of Janus nanoparticles in the strongly segregated lamellae phase of a diblock copolymer. In Section 3 we present our numerical results and Section 4 contains a detailed discussion. 


\section{MOLECULAR THEORY OF DIBLOCK COPOLYMER NANOCOMPOSITES}

In this section we consider a simple model of a rigid anisotropic nanoparticle composed of five equal spheres of radius $r_{0}$ separated by the distance $2 l$ (see Fig. 1). The interaction potential between the sphere $i(i=1-5)$ and the monomer unit $j$ of the type $\alpha(\alpha=A, B)$ is expressed as $V\left(r_{i j}\right)=J^{\alpha} r_{i j}^{-6}$ if $r_{i j}>r_{0}$ and $V\left(r_{i j}\right)=0$ if $r_{i j}<r_{0}$ where the coupling constant $J^{\alpha}$ depends on the type of the monomer unit $\alpha$ and $r_{0}$ is the radius of the sphere. Then the total interaction potential between a nanoparticle $p$ and all monomer units in the system is expressed as:

$$
V_{p}=\sum_{j} \sum_{i=1}^{5} J_{i, j}^{A}\left(r_{i p j}\right)+\sum_{j} \sum_{i=1}^{5} J_{i, j}^{B}\left(r_{i p j}\right),
$$

where $\mathbf{r}_{i p j}=\mathbf{r}_{p j}+\left(l+r_{0}\right)(i-3) \mathbf{a}_{p}$ and where $\mathbf{r}_{p j}$ is the vector between the centre of the nanoparticle $p$ and the monomer unit $j$. Here the unit vector $\mathbf{a}_{p}$ is along the axis of the nanorod $p$.

In the molecular field approximation, the one-particle distribution function is expressed as:

$$
f\left(\mathbf{a}_{p}, \mathbf{r}_{p}\right)=Z^{-1} \exp \left[-U_{M F}\left(\mathbf{a}_{p}, \mathbf{r}_{p}\right) / k_{B} T\right],
$$

where $Z$ is the normalization factor and the mean-field potential $U_{M F}\left(\mathbf{a}_{i}, \mathbf{r}_{i}\right)$ is given by the following expression:

$$
U_{M F}\left(\mathbf{a}_{p}, \mathbf{r}_{p}\right)=\int \sum_{i=1}^{5} J_{i, j}^{A}\left(r_{i p j}\right) \rho_{A}\left(\mathbf{r}_{j}\right) d^{3} \mathbf{r}_{j}+\int \sum_{i=1}^{5} J_{i, j}^{B}\left(r_{i p j}\right) \rho_{B}\left(\mathbf{r}_{j}\right) d^{3} \mathbf{r}_{j},
$$

where $\rho_{\alpha}\left(\mathbf{r}_{l}\right)$ is the density distribution of the monomer units $\alpha$. In the case of strong segregation $\rho_{\alpha}(\mathbf{r})=\rho_{0, \alpha}$ when $0<z<d_{\alpha}, \rho_{\alpha}(\mathbf{r})=0$ when $d_{\alpha}<z<d$, and $\rho_{\alpha}(z)=\rho_{\alpha}(z+d)$ for all $z$ as it is a periodic function with the period $d$. Here $d_{\alpha}$ is the thickness of the domain $\alpha$, the axis $z$ is perpendicular to the lamellae planes and $\rho_{0, \alpha}$ is the average density of monomer units $\alpha$ in the domain $\alpha$.

The mean-field potential for the whole NP is a sum of the potentials for all spheres constituting the nanorod. For a single sphere an explicit analytical expression for the meanfield potential has recently been obtained in Ref. [32]. In the case when the interaction range is much larger then $r_{0}$, the mean field potential for a single sphere is approximately expressed as:

$$
U_{M F i}(z)=\frac{\pi}{6} \Delta J z^{-3}-\frac{2 \pi}{3} \Delta J r_{0}^{-3}
$$


for $z>r_{0}$ and

$$
U_{M F i}(z)=-\frac{\pi z}{2} \Delta J r_{0}^{-4}
$$

for $0<z<r_{0}$ where $z$ is the distance between the centre of the sphere and the domain wall.

In the uniaxial lamellae phase the mean-field potential depends only on $z$ and $\cos \theta=$ $(\mathbf{a} \cdot \mathbf{h})$ where $\theta$ is the angle between the nanoparticle axis a and the unit vector $\mathbf{h}$. The total mean-field potential for the nanorod $p$ can now be written as a sum of five terms:

$$
U_{M F}(z, \theta)=\sum_{i=1}^{5} U_{M F i}\left(z_{i}\right)
$$

where $z_{i}=z+\left(l+r_{0}\right)(i-3) \cos \theta$ is the distance from the sphere "i" to the flat interface and where the potential $U_{M F i}\left(z_{i}\right)$ is given by the previous equation.

One notes that all $z_{i}$ depend both on $z$ and $\theta$ and as a result the inequalities $\left|z_{i}\right|<r_{0}$ and $\left|z_{i}\right|>r_{0}$, split the the plane $(z, \cos \theta)$ into numerous regions where the integration should be performed separately using variable limits. As shown in [34] even for a NP composed of two spheres the numerical integration can be dramatically simplified using the interpolated mean-field potential which is qualitatively valid for all values of $z$ and $\theta$.

$$
\begin{aligned}
U_{M F}^{*}(z, \theta)=-\frac{\pi}{6} \Delta J \tanh \left(z_{1}^{6} / r_{0}^{6}\right) z_{1}^{-3} & -\frac{2 \pi}{3} \Delta J \tanh \left(z_{1} / r_{0}\right) r_{0}^{-3} \\
& +\frac{\pi}{6} \Delta J \tanh \left(z_{2}^{6} / r_{0}^{6}\right) z_{2}^{-3}+\frac{2 \pi}{3} \Delta J \tanh \left(z_{2} / r_{0}\right) r_{0}^{-3},
\end{aligned}
$$

where $z_{1}=z+\left(l / 2+r_{0}\right) \cos \theta, z_{1}=z-\left(l / 2+r_{0}\right) \cos \theta$ and $\tanh \left(z / r_{0}\right)$ and $\tanh \left(z^{6} / r_{0}^{6}\right)$ are the interpolation functions.

This extrapolated potential can be generalised to the case of a nanorod composed of five equal interaction spheres:

$$
U_{M F}^{*}(z, \theta)=-\sum_{i=1}^{5}\left(\frac{\pi}{6} \Delta J \tanh \left(z_{1}^{6} / r_{0}^{6}\right) z_{1}^{-3}-\frac{2 \pi}{3} \Delta J \tanh \left(z_{1} / r_{0}\right) r_{0}^{-3}\right) .
$$

The local orientational order parameter $S(\mathbf{r})$ of the nanorods and the density distribution of nanoparticles can can now be written in the form:

$$
S(\mathbf{r})=\left\langle P_{2}\left(\mathbf{a}_{p} \cdot \mathbf{k}\right)\right\rangle=\frac{\int P_{2}\left(\mathbf{a}_{p} \cdot \mathbf{k}\right) f\left(\mathbf{a}_{p}, \mathbf{r}\right) d^{2} \mathbf{a}_{p}}{\int f\left(\mathbf{a}_{p}, \mathbf{r}\right) d^{2} \mathbf{a}_{p}},
$$

and

$$
\rho_{N}(\mathbf{r})=\rho_{N 0} \int f\left(\mathbf{a}_{i}, \mathbf{r}\right) d^{2} \mathbf{a}_{i}
$$


where $\rho_{N 0}$ is the average number density of nanoparticles and $P_{2}(x)$ is the second Legendre polynomial. Here the one-particle distribution function $f\left(\mathbf{a}_{i}, \mathbf{r}\right)$ is given by Eq.(2) where the mean-field potential is given by Eq.(6) for a NP composed of two spheres and by Eq.(7) for a NP composed of five spheres, respectively.

\section{LOCAL DENSITY AND ORIENTATIONAL ORDER PARAMETER PRO- FILES OF NANORODS}

One notes that in the boundary region, the mean-field potential depends on the NP position $z$ and on the orientation of the NP long axis a with respect to the boundary normal. One can readily see from Fig. 1 that in the boundary region the nanorods are orientationally ordered due to the discrepancy of interaction between the NP and the monomers of the two blocks. Indeed, let us assume that the interaction energy is minimised when all spheres of a nanorod are located in block B (see Fig.1). Thus one concludes that if the centre of a nanorod is in the block B close to the boundary, the nanorod is then aligned approximately parallel to the boundary because only in this case all interaction spheres are also located in the favorable block B. In contrast, if the nanorod centre is located in the block A, the nanorod axis is approximately normal to the boundary because in this case the maximum fraction of the rod is located in the favorable block B.

The orientational order parameter $S(z)$ and the local concentration of nanoparticles composed of five spheres have been calculated numerically and are presented in Figs. 2-6 where the origin $z=0$ is located at the boundary between the blocks. For comparison in Figs.2-6 we also present the order parameter and concentration distributions for shorter nanorods composed of the two spheres. One can readily see in Figs.2-3 that the main difference between the nanorods composed of five and two spheres, respectively, is in the width of the boundary region where the nanorods are orientationally ordered. Nanorods composed of five spheres are ordered in a significantly broader region which is an expected result related to the increase of the nanorod length. One notes that the effect of nanorod length can be separated from that of the increased number of the interaction sites by changing the separation $l$ between the spheres. The corresponding profiles are presented in Figs.4-6. Comparing the order parameter distributions for nanorods of different length (both with five interaction sites) presented in Fig.4 and Fig.5 enables one to conclude that the width of 
the boundary region (where the nanorods are orientationally ordered) is mainly determined by the length of the nanorod and not by the number of the interaction spheres. Moreover, the maximum value of the orientational order parameter is not very sensitive neither to the nanorod length nor to the number of interaction sites. In contrast, the order parameter is substantially increased with the increasing interaction constant $\Delta J$ between the NP and the monomer units (see Figs. 4,5).

\section{MOLECULAR DYNAMICS SIMULATIONS}

Simulations were performed using dissipative particle dynamics (DPD) for polymer systems [35] with the Groot-Warren thermostat [36] The simulation box of the three dimensions $l_{x} \times l_{y} \times l_{z}=24 \times 24 \times 24 r_{c}^{3}$ (where $r_{c}$ is a cut-off radius for interparticle interactions taken as the unit distance) is periodic in all three directions and has been filled with the total of 41472 DPD particles of three kinds: A, B, and (rod) R. A diblock copolymer chain $A_{10} B_{10}$ is composed of $N=20$ bonded particles forming the blocks $A$ and $B$ with $N_{A}=N_{B}=10$. Nanorods composed of $N_{R}=3,4$, or 5 particles connected by rigid bonds of the constant length $0.7 r_{c}$ have been simulated as rigid bodies in the $N V E$ ensemble using the Miller algorithm [37] The correct temperature of rods has been maintained through the interactions with the thermostated polymeric DPD liquid surrounding them. The equations of particle motion, $d \mathbf{r}_{i} / d t=\mathbf{v}_{i}, d \mathbf{v}_{i} / d t=\mathbf{F}_{i}$ have been solved numerically using a free source code LAMMPS [38] that implements the modified velocity-Verlet algorithm [39] with a time step $\delta t=0.02$. The repulsion parameter between identical particles, $a_{\alpha \alpha},(\alpha=A, B$, or $R$ )has been taken to be twice as large as the usual one [36] that was sufficient to achieve the nematic ordering in the pure nanorod melt. The interaction of nanorod particles with the blocks $\mathrm{A}$ and $\mathrm{B}$ is described by a selectivity parameter $\sigma=\left(a_{R B}-a_{R A}\right) /\left(a_{A B}-a_{R A}\right)$, which varies from zero for the non-selective case $\left(a_{R B}=a_{R A}=50\right)$ to unity in the case of maximum selectivity $a_{R B}=a_{A B}$. The overall volume fraction of nanorods, $\phi_{R}$, was varied from 0 to 0.1 , while the net volume fractions of $\mathrm{A}$ and $\mathrm{B}$ units were taken to be equal: $\phi_{A}=\phi_{B}=\left(1-\phi_{R}\right) / 2$. More details of the DPD simulation model can be found in ref. [33].

The simulations enable us to visualize stationary states of the composite and to describe them in terms of the local volume fractions of $\mathrm{A}, \mathrm{B}$, and $\mathrm{R}$ particles, $\phi_{A}(r), \phi_{B}(r)$, and 
$\phi_{R}(r)$, and the scalar orientational order parameter, $S=<\left[3(\mathbf{a}(\mathbf{r}) \cdot \mathbf{h})^{2} / 2-1 / 3\right]>$ which characterizes the average orientation of the nanorod axes $\mathbf{a}(|\mathbf{a}|=1)$ with respect to the selected direction $\mathbf{h}$. Here the angular brackets denote the averaging over a local subset of rods. In the lamellar microphase $\mathbf{h}$ is the unit vector normal to lamellar planes. Zero value of the order parameter corresponds to uncorrelated orientations of nanorods, whereas $S>0(S<0)$ specifies the orientational ordering in the direction perpendicular (parallel) to the lamellar plane. In general, $-0.5<S<1$. The profiles $\phi(z)$ and $S(z)$ are presented in Figs. 7 and 8, where the $z$-axis is parallel to $\mathbf{h}$ and the center of the layer B is chosen as the origin $(z=0)$.

One can readily see that the length of highly selective nanorods (at $\sigma=1$ they are composed of essentially the same DPD particles as the ones in the A copolymer blocks) has almost no effect on their local composition. This appears to be mainly defined by the balance of isotropic interactions between two kinds of monomer units and nanorods. The orientational ordering of nanorods is slightly more affected by the nanorod length. In particular, nanorods with $N_{R}=5$ demonstrate certain parallel alignment within their selective domain $\mathrm{A}$ and the absolute value of the orientational order parameter naturally decreases with the decreasing nanorod length.

For comparison we also present the order parameter and local concentration profiles of the so-called nonselective nanorods composed of spheres which interact exactly in the same way with monomer units of both blocks. One can readily see from Fig.7 that the local density of nanorods is significantly higher in the boundary region between the blocks and the nanorods are orientationally ordered throughout the lamellae phase which is rather unexpected. One may assume that sufficiently long nanorods have a tendency to phase separate at the boundary and maybe orientationally ordered for close packing reasons.

\section{DISCUSSION}

In this paper we have studied the orientational ordering and spatial distribution of nanorods of various lengths in the lamellae phase of diblock copolymers using both a molecular-statistical theory and dissipative particle dynamics simulations. The molecular theory is based on a simple model with fixed phase-separated structure of the diblock copolymer and a model of an anisotropic NP composed of two or five spheres which interact 
differently with the monomer units A and B. The effective interaction potential between such a nanorod and the monomer units appears to be anisotropic and as a result the nanorods are orientationally ordered in the boundary region where they interact simultaneous with the monomer units in both adjacent blocks. One notes that, in contrast to conventional liquid crystals, the orientational ordering of nanorods in real diblock copolymers is not expected to be self-consistent but is induced by the boundary between the blocks. The theory predicts that the orientational order parameter changes sign at the boundary that is the nanorods are aligned parallel to the boundary on one side of the domain wall and perpendicular to the boundary on another side. It has been shown that the width of the orientationally ordered region is increasing with the increasing nanorod length but is less sensitive to the number of interaction sites.

Computer simulations confirm the main qualitative conclusions of the molecular theory including the orientational order of nanorods and the sign inversion of the orientational order at the domain wall. However, there exist significant quantitative discrepancies. In particular, the absolute value of the orientational order parameter and the shape of the order parameter and concentration profiles differ from the ones predicted by the theory. The increase of the number of interaction spheres in the nanorod from two to five does improve the agreement between theory and computer simulations. However, the improvement is limited even in the case of nanorods composed of five spheres which closely mimic the NP used in simulations. For example, computer simulations indicate that the orientational order is not very sensitive to the molecular length and there is always some degree of orientational order in the bulk of the blocks. Thus the discrepancy between theory and dissipative dynamics simulations is most probably related to the approximations used in the theory including the model of the fixed distribution of the monomer units and the molecular-field approximation. Such a discrepancy is particularly obvious in the case of nonselective nanorods composed of the spheres which equally interact with the monomers A and B. In the context of the present simple theoretical model the discrimination interaction constant $\Delta J=0$ and hence the nanorods are not orientationally ordered and should be homogeneously distributed in the lamellae structure. In contrast, computer simulations indicate that a significant fraction of nonselective nanorods are located at the boundary and are orientationally ordered. This kind of ordering can be explained theoretically only taking into account explicitly the ordering of monomer units and the effect of NPs on their spatial distribution. Very recently such 
a molecular theory has been developed [40] which enables one to calculate self-consistently the spatial profiles of both the monomers and the nanorods.

\section{Acknowledgments}

The study was carried out within the state assignment of the Federal Agency for Scientific Organizations (FASO Russia) for TIPS RAS.

[1] Liquid Crystals with Nano and Microparticles, Lagerwall, J. P. F. and Scalia, G. (eds.). Singapore: World Scientific Publishing Company, (Series in Soft Condensed Matter; vol. 7)

[2] H. Qi, B. Kinkead, T. Hegmann,Effects of functionalized metal and semiconductor nanoparticles in nematic liquid crystal phases, Proc. SPIE 6911 (2008) 691106 (2008).

[3] H. Qi and T. Hegmann, "Formation of periodic stripe patterns in nematic liquid crystals doped with functionalized gold nanoparticles, J. Mater. Chem. 16 (2006) pp. 4197-4205.

[4] Y. Shiraishi, N. Toshima, K. Maeds, H. Yoshikawa, J. Xu, and S. Kobayashi:Frequency modulation response of a liquid-crystal electro-optic device doped with nanoparticles, Appl. Phys. Lett. 81 (2002) pp. 2845-2847.

[5] S.Kobayashi and N.Toshima,Nanoparticles and LCDs: It's a Surprising World, Information Display, 23 (2007) pp. 26-35.

[6] H. Yoshida, K. Kawamoto, H. Kubo, T. Tsuda, A. Fujii, S. Kuwabata, M. Ozaki,NanoparticleDispersed Liquid Crystals Fabricated by Sputter Doping, Advanced Materials 22 (2010) pp. $622-625$.

[7] P. Kopcansky, N. Tomasovicova, M. Koneracka, M. Timko, V. Zavisova, A. Dzarova, J. Jadzyn, E. Beaugnon, and X. Chaud, Phase Transitions in Liquid Crystal Doped with Magnetic Particles of Different Shapes, Int. J. Thermophys., 12 (2011) pp. 807-817.

[8] Yu.Reznikov, O.Buchnev, O.Tereshchenko, V.Reshetnyak, A.Glushchenko, and J.West, Ferroelectric Nematic Suspension, Appl. Phys. Lett.,82 (2003) pp. 1917-1919.

[9] F.Li, O.Buchnev, C.I. Cheon, A.Glushchenko, V.Reshetnyak, Yu.Reznikov, T.J.Sluckin, and J.L.West,Orientational Coupling Amplification in Ferroelectric Nematic Colloids, Phys.Rev.Lett. 97 (2006) 147801. 
[10] M.V. Gorkunov and M.A. Osipov, Mean-field theory of a nematic liquid crystal doped with anisotropic nanoparticles, Soft Matter, 7 (2011) pp. 4348-4356.

[11] O. Buchnev, A. Dyadyusha, M. Kaczmarek, V. Reshetnyak, Yu. Reznikov, J. Opt. Soc. Am. B 24 (2007) 1512.

[12] E. Ouskova, O. Bunchev, V. Reshetnyak and Yu. Reznikov, Dielectric relaxation spectroscopy of a nematic liquid crystal doped with ferroelectric Sn2P 2S6 nanoparticles Liq.Cryst. 30 (2003) pp. 1235-1239).

[13] S. Kaur, S. P. Singh, A. M. Biradar, A. Choudhary, and K. Sreeniva,Analog switching in the nanocolloids of ferroelectric liquid crystals Appl. Phys. Lett. 91 (2007) 023120.

[14] A. Kumar, J. Prakash, D. S. Mehta, A. M. Biradar, and W. Haase, Analog switching in the nanocolloids of ferroelectric liquid crystals, Appl. Phys. Lett. 95 (2009) 023117.

[15] E. B. Barmatov, D. A. Pebalk, M. V. Barmatova,Influence of silver nanoparticles on the order parameter of liquid crystalline polymers, Liq. Cryst. 33 (2006) pp. 1059-1063.

[16] P. Kopansk, N. Tomaoviov, M. Konerack, M. Timko, Z. Mitrov, V. Zviov, N. ber, K. FodorCsorba, T. Tth-Katona, A. Vajda, J. Jadzyn, E. Beaugnon, X. Chaud,Structural phase transition in liquid crystal doped with gold nanoparticles, Acta Phys. Polonica A, 118 (2010) pp. 988-989.

[17] G. Sinha, C. Glorieux and J. Thoen, Broadband dielectric spectroscopy study of molecular dynamics in the glass-forming liquid crystal isopentylcyanobiphenyl dispersed with aerosils, Phys. Rev. E, 69 (2004) 031707.

[18] T. Bellini, M. Buscagli, C. Chiccoli, F. Mantegazza, P. Pasini and C. Zannoni,Nematics with Quenched Disorder: What Is Left when Long Range Order Is Disrupted? Phys. Rev. Lett., $85(2000) 1008$.

[19] H. Duran, B. Gazdecki, A. Yamashita and T. Kyu,Effect of carbon nanotubes on phase transitions of nematic liquid crystals, Liq. Cryst. 32 (2005) pp. 815-821.

[20] L.M.Lopatina and J.R.Selinger, Maier-Saupe-type theory of ferroelectric nanoparticles in nematic liquid crystals, Phys. Rev. E, 84 (2011) 041703.

[21] M. Alexandre, P. Dubois, Polymer-layered silicate nanocomposites: preparation, properties and uses of a new class of materials. Mater. Sci. Eng. Rev. 28, (2000) pp. 1-32.

[22] A. C. Balazs, Interactions of nanoscopic particles with phase-separating polymeric mixtures, Curr. Opin. Colloid Interface Sci. 4, (2000) pp. 443-448. 
[23] E. P. Giannelis, Polymer-layered silicate nanocomposites: Synthesis, properties and applications, Appl. Organomet. Chem. 12, (1998) pp. 675-680.

[24] P. P. Soo, B. Y. Huang, Y. I. Jang, Y. M. Chiang, D. R. Sadoway, A. M. Mayes, Rubbery block copolymer electrolytes for solidstate rechargeable lithium batteries, J. Electrochem. Soc. 146, (1999) pp. 32-37.

[25] A. C. Balazs, T. Emrick, T. P. Russell, Nanoparticle polymer composites: where two small worlds meet, Science 314 (2006) pp. 1107-1110.

[26] R. Krishnamoorti, R. A. Vaia, Polymer Nanocomposites, J. Polym. Sci., Part B: Polym. Phys. 45, (2007) pp. 3252-3256.

[27] A. J. Crosby and J. Y. Lee, Polymer nanocomposites: the nano effect on mechanical properties, Polym. Rev. 47, (2007) pp.217-229.

[28] R.D. Deshmukh, Y. Liu, R.J. Composto,Two-Dimensional Confinement of Nanorods in Block Copolymer Domains, Nano Lett., 7 (2007) pp. 3662-3668.

[29] E. Ploshnik, A. Salant, U. Banin, R. Shenhar,Hierarchical Surface Patterns of Nanorods Obtained by Co-Assembly with Block Copolymers in Ultrathin Films, Adv. Mater.,22 ( 2010) pp. 2774-2779.

[30] E. Ploshnik, A. Salant, U. Banin, R. Shenhar,Co-assembly of block copolymers and nanorods in ultrathin films: effects of copolymer size and nanorod filling fraction, Phys. Chem. Chem. Phys., 12 (2010) pp. 11885-11893.

[31] K. Thorkelsson, A.J. Mastroianni, P. Ercius, T. Xu,Direct Nanorod Assembly Using Block Copolymer-Based Supramolecules, Nano Lett., 12 (2012) pp. 498-504.

[32] M.A. Osipov and M.V. Gorkunov,Spatial distribution and nematic ordering of anisotropic nanoparticles in lamellae and hexagonal phases of block copolymers, Eur. Phys. J. E, 39 (2016) pp. 126-134.

[33] A.V. Berezkin, Y.V. Kudryavtsev, M.V. Gorkunov, and M.A. Osipov, Ordering of anisotropic nanoparticles in diblock copolymer lamellae: Simulations with dissipative particle dynamics and a molecular theory, J. Chem. Phys., 146 (2017) 144902 .

[34] M. A. Osipov, A. S. Ushakova, M. V. Gorkunov, Orientational ordering of nanorods in diblock copolymers, Liquid Crystals, (2017) DOI: 10.1080/02678292.2017.1346826 .

[35] P. Espanol and P. B. Warren, Statistical mechanics of dissipative particle dynamics, Europhys. Lett. 30, 191 (1995). 
[36] 2. R. D. Groot and P. B. Warren, Dissipative particle dynamics: Bridging the gap between atomistic and mesoscopic simulation, J. Chem. Phys. 107, 4423 (1997).

[37] 3. T. F. Miller, M. Eleftheriou, P. Pattnaik, A. Ndirango, D. Newns and G. J. Martyna, Symplectic quaternion scheme for biophysical molecular dynamics, J. Chem. Phys. 116, 8649 (2002).

[38] 4. http://lammps.sandia.gov/

[39] 5. G. Besold, I. Vattulainen, M. Karttunen and J. M. Polson, Towards better integrators for dissipative particle dynamics simulations, Phys. Rev. E 62, R7611 (2000).

[40] M.A. Osipov, M.V. Gorkunov, A.V. Berezkin, and Y.V. Kudryavtsev, Phase behaviour and orientational ordering in block copolymers doped with anisotropic nanoparticles, Physical Review E. - in press 


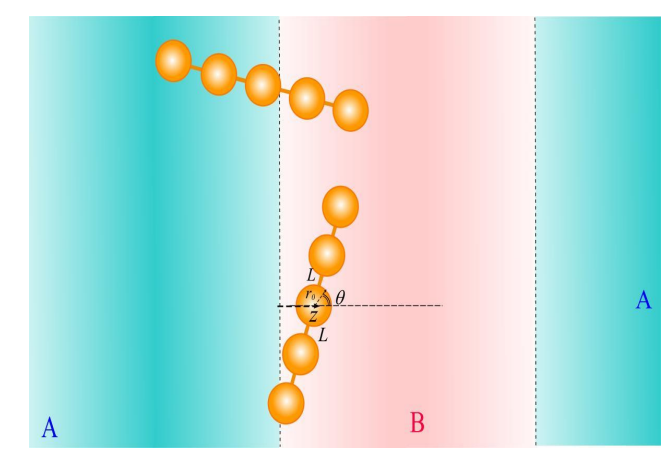

FIG. 1: Orientational ordering of anisotropic nanoparticles with five interaction centres in the boundary region between the blocks of the diblock-copolymer.

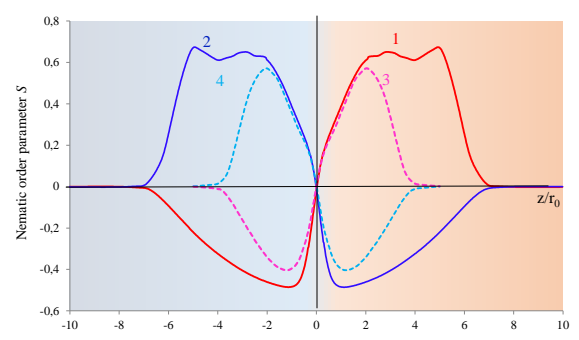

FIG. 2: Comparison of the orientational order parameter profiles of 5-unit (1-2) and 2-unit nanorods (3-4) in the lamellar phase of the diblock copolymer. The interaction constant $\Delta J=2 k_{B} T$ for the blue curves and $\Delta J=-2 k_{B} T$ for the red curves. Here $l=2 r_{0}$. 


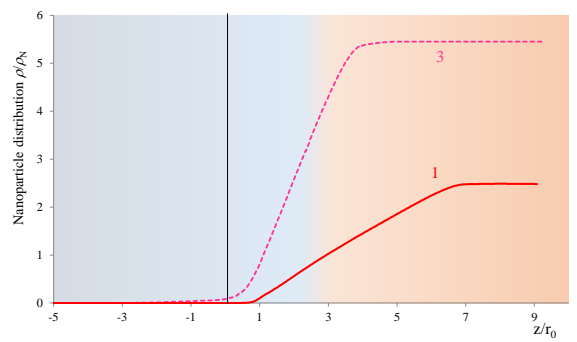

FIG. 3: Comparison of the local density profiles of 5-unit (1) and 2-unit nanorods (3) in the lamellar phase of the diblock copolymer. The interaction constant $\Delta J=2 k_{B} T$ and $l=2 r_{0}$.

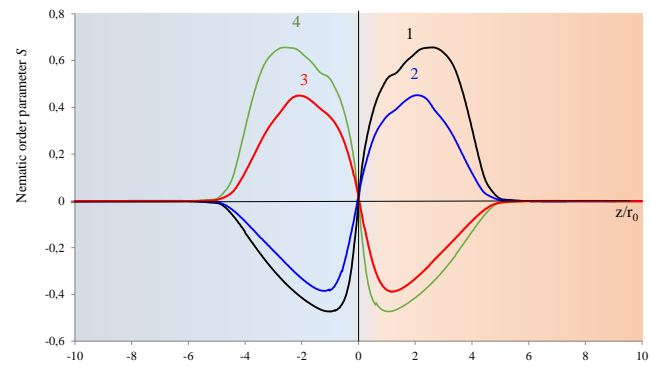

FIG. 4: Orientational order parameter profiles of shorter 5-unit nanorods with $l=r_{0}$ in the lamellae phase of the diblock copolymer for four different values of the interaction constant $\Delta J=$ $-2 k_{B} T(1),-k_{B} T(2), k_{B} T(3), 2 k_{B} T(4)$ 


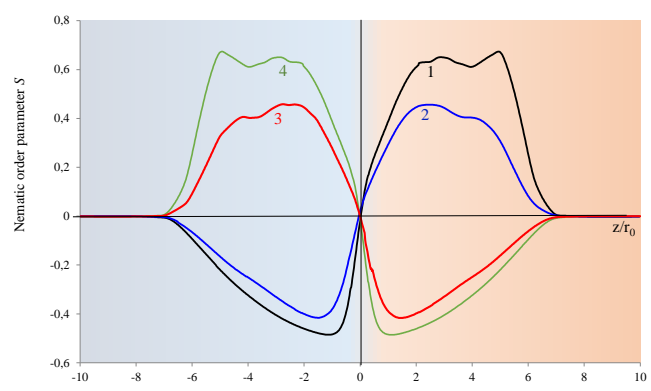

FIG. 5: Orientational order parameter profiles of longer 5-unit nanorods with $l=2 r_{0}$ in the lamellae phase of the diblock copolymer for four different values of the interaction constant $\Delta J=$ $-2 k_{B} T(1),-k_{B} T(2), k_{B} T(3), 2 k_{B} T(4)$

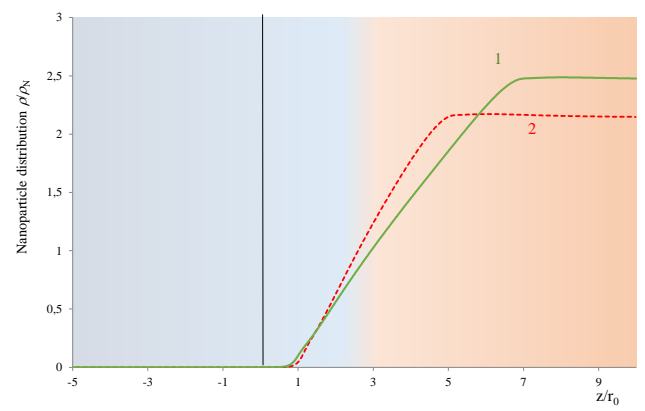

FIG. 6: Local density of longer 5-unit nanoparticles for $l=2 r_{0}(1)$ and $l=r_{0}(2)$ in the lamellar phase of a diblock copolymer. The interaction constant $\Delta J=2 k_{B} T$. 

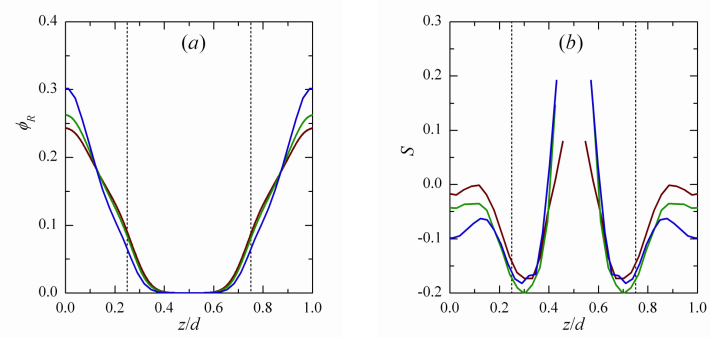

FIG. 7: Volume fraction profile $\phi_{R(z)}$ (a) and the orientational order parameter profile $S(z)$ (b) of nanorods composed of 3 (red), 4 (green), and 5 (blue) units. Vertical dashed lines show the domain boundaries in the pure diblock copolymer melt. In all cases $a_{A B}=a_{R B}=70, a_{R A}=$ $50(\sigma=1), \phi_{R}=0.1$.

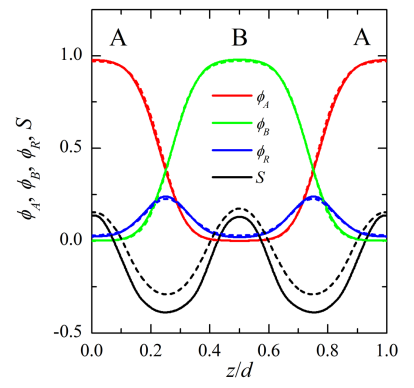

FIG. 8: Distribution of the monomer volume fraction, nanoparticle volume fraction and the orientational order parameter of the nanoparticles in the lamellar phase at $N_{R}=5$ (dashes) and 7 (solid lines) where $\sigma=0$. 